\title{
Utilisation d'une chambre de transpiration portable pour l'estimation de l'évapotranspiration d'un sous-bois de pin maritime à molinie (Molinia coerulea (L) Moench)
}

\author{
D Loustau *, H Cochard \\ Avec la collaboration technique de M Sartore et M Guédon \\ INRA, Recherches forestières, laboratoire d'écophysiologie et nutrition, \\ domaine de l'Hermitage BP 45, 33610 Cestas, France
}

(Reçu le 6 avril 1990; accepté le 15 octobre 1990)

\begin{abstract}
Résumé - Une chambre de transpiration légère, close, couvrant une surface au sol de $0,5 \mathrm{~m}^{2} \mathrm{a}$ été utilisée pour mesurer l'évapotranspiration (ET) de touffes de molinie en sous-bois d'un peuplement de 19 ans de pin maritime. L'ET horaire et journalière du sous-bois et sa conductance de couvert ont été estimées au cours de 13 journées de mesure réalisées sur 2 saisons de croissance en 1988 et en 1989. Ces mesures sont comparées avec la transpiration des arbres estimée à partir de mesures de flux de sève. Quand le stock hydrique du sol est supérieur à $40 \mathrm{~mm}$, l'évapotranspiration de la molinie est comprise entre 1 et $1,5 \mathrm{~mm} / \mathrm{j}$. Elle correspond à $1 / 3$ de celle des arbres et représente une fraction constante de l'ETP à $1 \mathrm{~m}$. La conductance de couvert du sous-bois est globalement constante pendant la journée et varie entre 0,4 et $0,5 \mathrm{~cm} \cdot \mathrm{s}^{-1}$. Au cours de l'été, quand le stock hydrique diminue en dessous de $40 \mathrm{~mm}$, l'évapotranspiration et la conductance de couvert de la molinie diminuent d'un facteur 2-3. La transpiration du pin maritime présente un comportement différent : elle est réduite dès que le stock descend en dessous de $55 \mathrm{~mm}$, et dans des proportions plus importantes.
\end{abstract}

conductance de colvert / sécheresse édaphique / indice foliaire / variation saisonnière

Summary - Use of a portable transpiration chamber for estimating the evapotranspiration of the understory of a maritime pine stand. Water stress is frequent in stands of maritime pine in Southwest France and silvicultural practices should be optimized with this factor in mind. The consumption of available water by the vegetation of the understory has not been quantified, though it should be taken into account in management practices such as thinning or fertilization. This paper reports the results of measurements of hourly and daily evapotranspiration (ET) of a 19-yr-old maritime pine (Pinus pinaster Ait) stand understory. A portable closed transpiration chamber covering a projected area of $0.5 \mathrm{~m}^{2}$ was used to measure the ET from purple heath grass (Molinia coerulea $(L)$ Moench) which accounted for more than $90 \%$ of the leaf area index of the understory. The ET was determined by enclosing tussocks and measuring the variations of dew-point temperature for 1 min. From these measurements, both the daily and hourly ET of the understory was estimated for $13 \mathrm{~d}$ in 1988 and 1989. The canopy conductance of the understory was also estimated. These results were compared with the transpiration of pine computed from sap flow measurements. When the total

\footnotetext{
* Correspondance et tirés à part.
} 
water content of the rooted zone was above $40 \mathrm{~mm}$, the ET of the understory varied between 1 and $1.5 \mathrm{~mm}^{\circ} \mathrm{day}^{-1}$; it remained constant in relation to the Penman PET measured at $1 \mathrm{~m}$ above the ground. This corresponded to $1 / 3$ of the transpiration of the trees. Understory canopy conductance remained fairly constant throughout the day and fluctuated between 0.4 and $0.5 \mathrm{~cm} \cdot \mathrm{s}^{-1}$. When the soil water content dropped below $40 \mathrm{~mm}$, the $\mathrm{ET}$ and the canopy conductance of the understory were reduced by a factor of 2-3. The transpiration of pines was more sensitive to soil water deficit and was reduced by a factor of 4 to 6 when the soil water content dropped below $55 \mathrm{~mm}$.

canopy conductance / soil water deficit / leaf area index / seasonal change

\section{INTRODUCTION}

La sécheresse édaphique est un facteur limitant de la production ligneuse commun à l'ensemble des forêts de production à sylviculture intensive sous climat de type méditerranéen (forêts de Pinus radiata, Eucalyptus, Pinus pinaster, Pinus taeda, etc). Elle limite également la.production forestière sous des climats plus océaniques comme dans le cas du douglas dans l'Ouest canadien (Black et al, 1980).

La connaissance de la contribution de la végétation du sous-étage des arbres au bilan hydrique des peuplements, est nécessaire pour optimiser la conduite de ces peuplements vis-à-vis de cette contrainte. Cependant, il existe peu de travaux concernant l'estimation et la modélisation de l'évapotranspiration des sous-étages forestiers.

Lindroth (1984) a évalué la transpiration d'un sous-bois de pin sylvestre par lysimétrie. Tan et al (1978) ont estimé la transpiration d'un tapis d'éricacées (Gaultheria shallon Pursh) sous un couvert de douglas à partir d'un modèle de diffusion de vapeur simple, utilisant le déficit de saturation de l'air et la conductance stomatique des espèces du sous-bois. Roberts et al (1980) ont utilisé la relation de PenmanMonteith en estimant une conductance de couvert à partir de mesures porométriques et une conductance aérodynamique à partir d'une mesure d'évaporation de feuilles humides. Par cette méthode, les variations saisonnières de transpiration d'un sousbois de pin sylvestre à fougères ont été estimées au cours d'une saison de végétation. Kelliher et al (1986) ont proposé une modélisation de la transpiration d'un sousétage de douglas à partir d'un modèle d'évapotranspiration à plusieurs couches fondé sur la relation de Penman-Monteith.

Ces travaux concernent surtout des forêts soumises à un climat plus océanique ou plus froid, et où la sécheresse édaphique est moins fréquente que sous les climats de type méditerranéen.

Par ailleurs, même si les résultats présentés sont le plus souvent vérifiés par d'autres méthodes indépendantes, ces modèles reposent le plus souvent sur un échantillonnage spatial restreint de mesures porométriques ou lysimétriques.

Bien qu'elles soient utilisées pour l'estimation de l'ET et de la photosynthèse de couverts végétaux de plantes annuelles (Decker et al, 1962; Peters et al, 1974; Reicosky et Peters, 1977; Daudet, 1987), les méthodes de chambre d'assimilation et de transpiration sont peu développées sur des sous-bois forestiers. Elles ont l'avantage de fournir une mesure intégrant l'ET d'un couvert végétal sur une surface de plusieurs $\mathrm{dm}^{2}$ à plusieurs $\mathrm{m}^{2}$ suivant les cas. Mais la plupart des systèmes utilisés sont assez encombrants et souvent fixes.

La variabilité spatiale de la végétation et des conditions microclimatiques en sous- 
bois requiert au contraire la possibilité d'échantillonner les mesures effectuées.

Dans le cadre d'un programme d'étude du fonctionnement hydrique d'un peuplement de pins maritimes (Diawara et al, 1990; Loustau et al, 1990), un système de chambre de transpiration léger et mobile a été mis au point pour la mesure de l'ET du sous-étage. Cet article présente le principe et la réalisation de ce système, et les résultats d'une estimation de l'ET et de la conductance de couvert du sous-étage, obtenus à partir des mesures effectuées.

\section{MATÉRIEL ET MÉTHODES}

\section{Site expérimental}

Les principales caractéristiques du site du Bray $\left(44^{\circ} 42^{\prime} \mathrm{N}, 0^{\circ} 46^{\prime} \mathrm{W}\right)$ ont été décrites par ailleurs (Loustau et al, 1990).

La parcelle expérimentale est située au sein d'un massif de plusieurs centaines d'hectares. Elle couvre 16 ha d'un seul tenant. Le placeau où ont été opérées les différentes mesures présentées ci-après couvre une superficie de 0,25 ha au centre de la parcelle.

Le peuplement végétal est structuré en 2 strates qui présentent une grande homogénéité spatiale :

- une strate arborescente constituée de pins maritimes (Pinus pinaster Ait) alignés en rangs espacés de $4 \mathrm{~m}$, issus d'un semis de 1970;
- une strate herbacée dont la molinie (Molinia coerulea L Moench) représente plus de $90 \%$ de l'indice foliaire à plein développement et qui compend également les espèces suivantes : Pseudarrhenaterum longifolium, Ulex europaeus, Erica scoparia et d'autres espèces dont la contribution à l'indice foliaire total de cette strate est négligeable.

La molinie est une graminée à rhizome, cespiteuse, à croissance sympodiale. Son cycle annuel présente une phase de développement végétatif comprenant le développement et la croissance de 3-4 feuilles, et une phase reproductrice (montaison, floraison, épiaison) qui précède la fanaison automnale. Elle constitue en sous-bois un tapis de touffes juxtaposées, plus ou moins bien délimitées, constituées chacune de centaines de tiges. C'est l'espèce la plus importante des milieux de "lande humide", principale unité écologique du massif forestier des Landes de Gascogne.

Le tableau I présente les principales caractéristiques physiques des 2 strates végétales du site.

Le sol de la parcelle est un podzol humique à alios caractérisé par la présence à une profondeur moyenne de $65 \mathrm{~cm}$ d'un horizon d'accumulation de sesquioxydes métalliques amorphes ( $\mathrm{Fe}$ et $\mathrm{Al}$ ), induré, qui limite l'extension en profondeur des racines de molinie, de pin et des autres végétaux.

A cours des étés 1988 et 1989, une sécheresse édaphique s'est développée et a provoqué une réduction de la transpiration des pins à partir de septembre en 1988 et de juillet en 1989 (Loustau et al, 1990).

Tableau I. Principales caractéristiques physiques du peuplement.

\begin{tabular}{lccccc}
\hline & $\begin{array}{c}\text { Hauteur } \\
\text { moyenne }(m)\end{array}$ & $\begin{array}{c}\text { Circonférence } \\
\text { moyenne }(m)\end{array}$ & $\begin{array}{c}\text { Densité } \\
\text { (Tiges/ha) }\end{array}$ & $\begin{array}{c}\text { Biomasse } \\
\text { (tms/ha) }\end{array}$ & Indice foliaire \\
\hline $\begin{array}{l}\text { Strate arborescente } \\
\text { (pin maritime) }\end{array}$ & 12 & 0,60 & 850 & 80 & 3 \\
$\begin{array}{l}\text { Strate herbacée } \\
\text { (molinie) }\end{array}$ & 0,6 & - & - & 1 & 1,2 \\
\hline
\end{tabular}




\section{Mesure de l'évapotranspiration et calcul de conductance}

\section{Principe}

Le taux de diffusion de vapeur, ou évaporation, $E$ en $\left(\mathrm{kg} \cdot \mathrm{m}^{-2} \cdot \mathrm{s}^{-1}\right)$ à partir d'une surface évaporante humide est donné par :

$$
E=g_{w} \frac{M_{\mathrm{H}_{2} \mathrm{O}} \cdot \rho}{M_{\mathrm{air}} \cdot P}\left(e s_{(\tau s)}-e s_{(\tau r)}\right)
$$

avec :

$g_{w}=$ conductance à la diffusion de vapeur entre la surface et l'atmosphère $\left(\mathrm{m} . \mathrm{s}^{-1}\right)$,

$\rho=$ masse volumique de l'air sec $\left(\mathrm{kg} \cdot \mathrm{m}^{-3}\right)$,

$\mathrm{M}_{\mathrm{H}_{2} \mathrm{O}}=$ masse molaire de l'eau $(\mathrm{kg})$,

$M_{\text {air }}=$ masse molaire effective de l'air $(\mathrm{kg})$,

$P=$ pression atmosphérique $(\mathrm{Pa})$,

$e s_{(T s)}=$ pression de vapeur saturante $(\mathrm{Pa})$ à la température de la surface évaporante,

$e s_{(T r)}=$ pression de vapeur saturante $(\mathrm{Pa})$ à la température de rosée de l'air.

Dans le cas d'une feuille, on admet classiquement que la pression de vapeur dans le mésophylle est saturante. Si on néglige la différence entre la température de la feuille et celle de l'air (cette hypothèse sera discutée plus loin), ron a alors :

$$
\begin{aligned}
& e s_{(T s)}=e s_{(T a)} \\
& E=g_{w} \cdot \frac{M_{\mathrm{H}_{2} O} \rho}{M_{\mathrm{air}} \cdot P}\left(e s_{(T a)}-e s_{(T r)}\right)
\end{aligned}
$$

Par intégration, on montre que pour une plante placée dans une enceinte close idéalement isotherme, la quantité de vapeur d'eau contenue dans le volume de la cage et la conductance $\mathrm{g}_{w^{\prime}}$ sont liées par la relation :

$$
q w_{t}=\left(q w_{0}-q w_{s}\right) \cdot e^{-\frac{t(q w)}{v}}+q w_{s}
$$

$q w_{t}=$ quantité totale de vapeur d'eau contenue dans le volume de l'enceinte à l'instant $t(\mathrm{~kg})$, $q w_{0}=$ quantité de vapeur d'eau initialement présente $(\mathrm{kg})$, $q w_{s}=$ quantité de vapeur à saturation $(\mathrm{kg})$, $t=$ durée écoulée depuis l'instant initial (s), $v=$ volume de l'enceinte $\left(\mathrm{m}^{3}\right)$.

La transpiration de la plante est la dérivée de cette fonction à l'instant $t=0$.

La conductance de la surface évaporante à la diffusion de vapeur $g_{w}$, peut s'exprimer en fonction de $E$ à partir de l'équation (2):

$$
g_{w}=\frac{E \cdot M_{\mathrm{air}} P}{M_{\mathrm{H}_{2} \mathrm{O}} \cdot \rho\left(e s_{\left(\mathrm{Ta}_{\mathrm{a}}\right)}-e s_{(\mathrm{Tr})}\right)}
$$

$g_{w}$ correspond à la conductance équivalente à 2 conductances en série, la conductance de surface $g_{s}$ et la conductance aérodynamique $g_{a}$ :

$$
\frac{1}{g_{w}}=\frac{1}{g_{s}}+\frac{1}{g_{a}}
$$

\section{Application}

La calibration du système de mesure utilisé a montré que la courbe d'augmentation de $q w(t)$ est pratiquement linéaire durant les 2 premières minutes consécutives au confinement d'une surface évaporante par la fermeture de la chambre, et ceci pour une large gamme de taux d'évaporation. La dérivée à l'origine de $q w_{t}$ (équation (3)) a donc été assimilée à la pente de cette droite et donne le taux d'évapotranspiration en $\mathrm{kg} \cdot \mathrm{s}^{-1}$. Ce taux est exprimé en $\mathrm{mm}$ sur la base de la surface au sol de la chambre.

Durant ce court laps de temps, l'augmentation de température enregistrée est typiquement de $0,3^{\circ} \mathrm{C}$ et n'excède jamais $0,5^{\circ} \mathrm{C}$.

Nous avons défini la conductance de couvert $(g)$ de la plante comme la conductance équivalente globale de la plante introduite dans la chambre (Cruiziat et al, 1979). En utilisant les valeurs d'évapotranspiration mesurées, la conductance de couvert a été calculée à partir des équations (4) et (5) avec une valeur approchée de la conductance aérodynamique $g_{a}$ Cette approximation a été opérée comme suit :

- les vitesses de vent mesurées avec un anémomètre à coupelles en divers points de la chambre varient entre 0,5 et $1,0 \mathrm{~m} . \mathrm{s}^{-1}$;

- le nombre de Reynolds calculé dans cette gamme de vitesse de vent pour des feuilles de 
molinie est autour de $2.10^{3}$, ce qui correspond à la transition entre convection laminaire et convection turbulente.

Suivant l'hypothèse d'une convection laminaire, $g_{a}$ peut être estimée pour un ensemble de feuilles en interférence mutuelle (Landsberg et Powell, 1973) par :

$$
g_{a}=\quad\left[195 \cdot\left(\frac{d}{u}\right)^{0,5}\right]^{-1}
$$

avec :

$g_{a}:$ conductance aérodynamique $\left(\mathrm{m}^{\mathrm{s}} \mathrm{s}^{-1}\right)$, $u$ : vitesse du vent $\left(\mathrm{m} . \mathrm{s}^{-1}\right)$,

$d$ : dimension caractéristique de la feuille, qui, dans le cas d'une feuille de graminée, correspond approximativement aux $9 / 10$ de sa largeur (m).

Pour des vitesses de vent allant de 0,25 à $1,00 \mathrm{~m} . \mathrm{s}^{-1}$ et des feuilles de largeur moyenne de $6 \mathrm{~mm}$, cette approximation conduit à des valeurs de $g_{a}$ comprises entre 0,025 et 0,075 m.s ${ }^{-1}$. En régime de convection turbulente les valeurs de $g_{a}$ seraient multipliées par un facteur compris entre 1 et 2 (Jones, 1983). Nous avons fixé empiriquement la valeur de $g_{a}$ à $0,050 \mathrm{~m} . \mathrm{s}^{-1}$, ce qui correspond à la conductance de la couche limite d'une culture haute ventilée et $g_{c}$ a été calculée par :

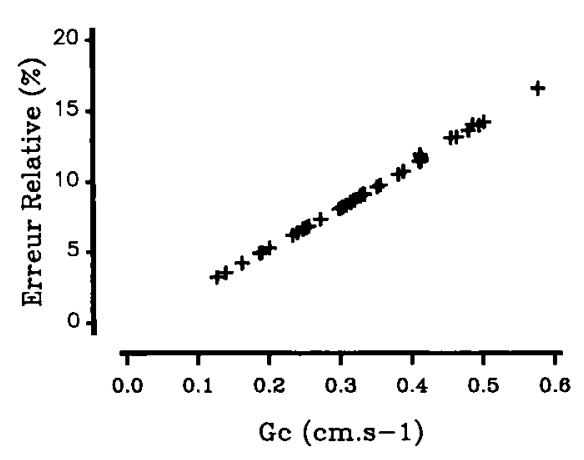

Fig 1. Erreur relative maximale induite sur l'esti-i mation de la conductance de couvert par l'approximation d'une valeur de conductance de la couche limite, en fonction des valeurs de la conductance de couvert.
1

$$
g_{c}=\frac{M_{\mathrm{H}_{2} \mathrm{O}} \cdot \rho \cdot\left(e s_{(\mathrm{Ta})}-\theta s_{(T r)}\right)}{M_{\mathrm{air}} \cdot E \cdot P}-\frac{1}{0,05}
$$

Dans la suite de cet article, les valeurs de conductance ont été exprimées en $\mathrm{cm} \cdot \mathrm{s}^{-1}$.

Afin d'évaluer la sensibilité du calcul de $g_{c}$ aux erreurs commises sur $g_{a}$, nous avons testé l'effet de variations de valeurs de $g_{a}$ sur les valeurs calculées de $g_{c}$

Sur un échantillon de 30 mesures couvrant la gamme des valeurs de $g_{c}$ mesurées au cours des 2 années d'expérimentation, nous avons calculé les valeurs de $g_{c 2,5}, g_{c 5,0}$ et de $g_{c 7,5}$ correspondant respectivement aux valeurs de $g_{c}$ calculées avec $g_{a}=2,5 \mathrm{~cm} . \mathrm{s}^{-1}, g_{a}=5 \mathrm{~cm} . \mathrm{s}^{-1}$ et $g_{a}=7,5 \mathrm{~cm} \cdot \mathrm{s}^{-1}$. Considérant que les valeurs possibles de $g_{a}$ étaient comprises entre 2,5 et $7,5 \mathrm{~cm} . \mathrm{s}^{-1}$, le rapport $\left(g_{c 2,5}-g_{c 7,5}\right) / g_{c 5}$ donne l'enveloppe supérieure des erreurs relatives induites dans le calcul de $g_{c}$ par l'approximation commise sur $g_{a}$. La figure 1 représente les valeurs de l'erreur relative maximimale pour l'échantillon extrait. L'erreur varie entre 0 et $20 \%$ et augmente de façon presque linéaire en fonction de $g_{c 5}$. Ceci doit donc conduire à une certaine réserve quant à linterprétation des données d'évapotranspiration mesurées avec cette chambre en terme de conductance, particulièrement pour les fortes valeurs de celle-ci.

\section{Description du système de mesure et protocole des mesures}

Deux cages psychrométriques de même surface $(0,76 \times 0,76 \mathrm{~m})$ mais différentes par leur hauteur $(0,92$ et $0,46 \mathrm{~cm}$ respectivement) ont été utilisées, en fonction de la hauteur de la végétation.

Elles sont constituées par une armature en aluminum (tubes de section carrée de $0,02 \times$ $0,02 \mathrm{~m}$ ) couverte d'un film en polypropylène de $28 \mu \mathrm{m}$ d'épaisseur. Le prolypropylène possède une faible perméabilité à la vapeur d'eau $(22,2$ $\mathrm{nmol} . \mathrm{mm}^{2} \cdot \mathrm{s}^{-1} \mathrm{~Pa}^{-1}$ ) et une capacité d'absorption de la vapeur d'eau négligeable (inférieure à 2 mmol. $\mathrm{kg}^{-1} . \mathrm{j}^{-1}$ ).

Le brassage d'air dans l'enceinte est assuré par 2 ventilateurs axiaux, (Etri - réf DTM $125+2$, 21000 ) qui pulsent l'air de la chambre avec un débit de $20-25 \mathrm{dm}^{3} . \mathrm{s}^{-1}$. Un hygromètre à point 


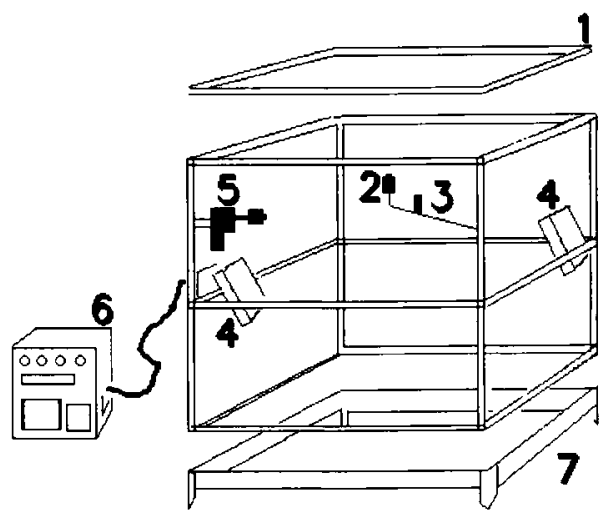

Fig 2. Schéma général de la chambre. 1 : couvercle, 2 : pyranomètre, 3 : sonde PT 100; 4 : ventilateurs; 5 : hygromètre à point de rosée; 6 : boîtier de commande, incluant batteries et centrale d'acquisition; 7 : socle enterré.

de rosée (transmetteur Dew10, General Eastern) donne la température de rosée de l'air.

La température de l'air est mesurée avec une sonde PT 100 (Pyrocontrôle réf 31-001110) placée à proximité de l'hygromètre. L'irradiance énergétique reçue au centre de la chambre est mesurée par un pyranomètre à cellule de silicium (Skye Instruments). Le schéma général du système est donné dans la figure 2 .

La température, l'hygrométrie et l'irradiance énergétique sont mesurées toutes les $0,5 \mathrm{~s}$ et les moyennes des 4 mesures consécutives sont enregistrées par une Centrale Campbell 21X. Le déficit de saturation $(\mathrm{Pa})$ est calculé à partir de la température de rosée, $\operatorname{Tr}\left({ }^{\circ} \mathrm{C}\right)$ par :

$$
o_{s a t}=e s_{(T a)}-e_{\left(T_{a}\right)}=e s_{(T a)}-e s_{(T r)}
$$

es $(T r)$ étant calculé en fonction de la température $T$ selon l'algorithme de Murray (1967).

L'alimentation électrique (24 V) est fournie par 2 batteries légères de $12 \mathrm{~V}$ en série. Ce dispositif est autonome et maniable par un seul expérimentateur.

Chaque mesure dure de $30-60 \mathrm{~s}$. Une droite de régression est ajustée à la courbe d'augmentation $q w=f(t)$ (Proc Reg Logiciel SAS). L'évapotranspiration dans la chambre, exprimée par unité de surface du sol $\left(\mathrm{mm} . \mathrm{s}^{-1}\right)$, est donnée par la pente de cette droite. Les mesures pour lesquelles le coefficient de détermination $r^{2}$ de l'ajustement est inférieur à 0,95 sont rejetées (cela correspond à 2-3\% des cas rencontrés).

La conductance de couvert, $g c\left(\mathrm{~cm}^{-1}\right)$, est ensuite calculée suivant l'équation (6).

\section{Estimation des moyennes horaires du taux d'évapotranspiration et de la conductance de surface en sous-bois}

Lors de chaque journée de mesure, 10 touffes de molinie ont été choisies au hasard, au milieu de la parcelle expérimentale. Chaque touffe était entourée d'un socle carré en plexiglas de même dimension au sol que la chambre et de $8 \mathrm{~cm}$ de haut, enfoncé dans le sol sur une profondeur de $2-3 \mathrm{~cm}$, destiné à assurer l'étanchéité de l'enceinte. Des mesures de l'ET de chaque touffe ont été répétées au cours de la journée à raison d'une par heure environ, du matin au soir.

En 1989, la moitié des touffes de l'échantillon était coupée à la fin de la journée pour la mesure de leur surface foliaire.

L'évapotranspiration et la moyenne horaire de conductance de couvert du sous-bois ont été estimées par la moyenne arithmétique des mesures opérées durant le même intervalle horaire. L'évapotranspiration journalière a été estimée par la somme des produits entre l'ET horaire $\left(m m \cdot h^{-1}\right)$ et la durée de l'intervalle horaire $(e n h)$ considéré.

\section{Autres mesures}

\section{L'évapotranspiration horaire et journalière des arbres}

Elle a été calculée à partir des valeurs de flux de sève mesuré par une méthode thermoélectrique à flux de chaleur continue sur un échantillon de 10 arbres (Granier, 1987; Loustau et al, 1990). 


\section{L'ETP journalière au-dessus du peuplement}

Elle a été estimée par la formule de Penman à partir de mesures micrométéorologiques opérées à partir de capteurs de température de l'air, de vitesse de vent, de rayonnement global et d'hygrométrie placés sur un échafaudage $16 \mathrm{~m}$ de haut (Loustau et al, 1990).

\section{L'ETP à $1 \mathrm{~m}$ au-dessus du sol}

Elle a été calculée selon la relation de Penman, à partir des mesures de température, d'hygrométrie et d'irradiance énergétique opérées au cours des mesures d'ET du sous-bois avec la chambre de transpiration :

$$
E T P=\frac{s \cdot A+\rho \cdot C p \cdot h \cdot D_{\mathrm{sat}}}{L \cdot(s+\gamma)}
$$

avec:

$A=$ énergie disponible $\left(W . \mathrm{m}^{-2}\right)$,

$D_{\text {sat }}=$ déficit de saturation de l'air $(\mathrm{Pa})$,

$\mathrm{s}=$ dérivée de la fonction reliant la pression de vapeur saturante à la température $\left(\mathrm{Pa}^{\circ} \mathrm{C}^{-1}\right)$,

$L=$ chaleur latente de vaporisation de l'eau $\left(\mathrm{J} . \mathrm{kg}^{-1}\right)$,

$\gamma=$ constante psychrométrique $\left(\mathrm{Pa} .{ }^{\circ} \mathrm{C}^{-1}\right)$,

$p=$ masse volumique de l'air sec $\left(\mathrm{kg} \cdot \mathrm{m}^{-3}\right)$,

$h=$ coefficient d'échange convectif $\left(\mathrm{m}^{-\mathrm{s}^{-1}}\right)$.

La quantité d'énergie disponible a été fixée empiriquement comme une fraction constante de l'irradiance énergétique à $1 \mathrm{~m}: A=0,75, R g$. Une valeur moyenne de conductance aérodynamique de $0,025 \mathrm{~m} . \mathrm{s}^{-1}$, estimée à partir de mesures du flux de chaleur sensible à $1 \mathrm{~m}$ (Amadou, 1989; Berbigier et al, 1990), a été assignée au coefficient d'échange convectif, $h$.

\section{Le stock hydrique du sol dans la sphère racinaire $(0-75 \mathrm{~cm})$}

II a été calculé à partir des mesures neutroniques de l'humidité volumique opérées tous les 10 j environ, sur un réseau de 8 tubes d'accès neutroniques, à raison d'une mesure par tranche de $10 \mathrm{~cm}$.

\section{L'indice foliaire de la molinie}

II a été estimé 1 fois en fin de saison en 1988 et à 5 reprises en 1989. La biomasse sèche moyenne, $B\left(\mathrm{~kg} / \mathrm{m}^{2}\right)$, est estimée par la moyenne arithmétique d'un échantillon de 20 mesures. Chaque mesure correspond à un placeau de $0,5 \times 0,5 \mathrm{~m}$ dont la végétation est fauchée, séchée à $65^{\circ} \mathrm{C}$ à poids constant et pesée. La surface massique moyenne, $S n\left(\mathrm{~m}^{2}\right)$ $\mathrm{kg}$ ), des feuilles de molinie est évaluée par la moyenne arithmétique de surfaces massiques d'un échantillon aléatoire de 20 feuilles. La surface totale ( 1 face) de chaque feuille est mesurée avec un planimètre (Delta $T$ ) et son poids sec mesuré suivant le même protocole que précédemment.

L'indice foliaire est donné par :

$$
I F=\operatorname{Sn} \times B .
$$

L'évolution phénologique a été observée toutes les semaines, en 1988 comme en 1989 , sur un échantillon de 100 tiges réparties sur 6 touffes différentes.

\section{RÉSULTATS}

\section{Phénologie et indice foliaire}

Les premières feuilles en croissance sont apparues en 1988 et en 1989, au début du mois de mars. La succession des stades phénologiques en 1988 s'est faite de façon plus tardive qu'en 1989 , le décalage entre ces 2 années allant de 15 j à 3 semaines environ. En 1989, la $2^{e}$ feuille est apparue fin avril et la $3 e$ vers la fin mai. Moins de $10 \%$ des tiges ont développé une $4^{\theta}$ feuille. La montaison a commencé dans la $2^{\mathrm{e}}$ quinzaine de juin; floraison et épiaison se sont ensuite succédé en juillet et août. La fanaison des feuilles a été observée à partir de septembre et s'est terminée en novembre.

En 1989, l'indice foliaire a crû rapidement entre avril et fin mai où il a dépassé 


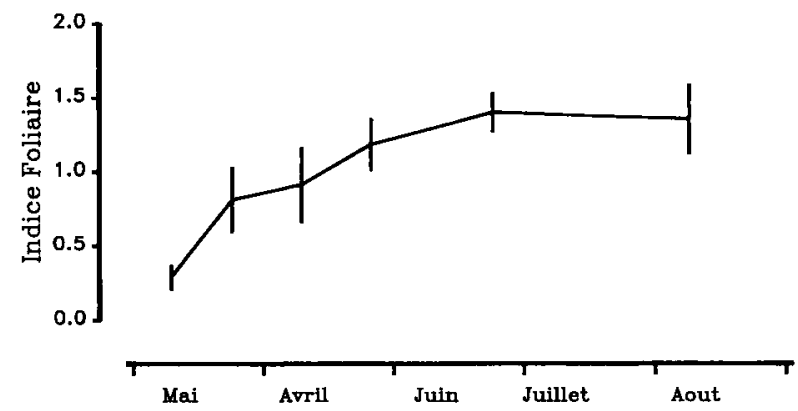

Fig 3. Évolution de l'indice foliaire de la molinie en 1989 (les barres verticales représentent l'intervalle de confiance à $95 \%$ de la valeur moyenne estimée).

la valeur 1 (fig 3 ). L'indice foliaire maximal est atteint vers la fin du mois de juin. II est compris entre 1,2 et 1,5. L'indice foliaire ne varie plus de façon importante jusqu'au mois d'octobre où commence la fanaison qui s'achève vers la mi-novembre. Ce sont les $2^{e}$ et $3^{e}$ feuilles qui contribuent le plus

Fig 4. Évolution journalière de l'ETP à $1 \mathrm{~m}$ en sous-bois, de l'ET du sous-étage (graphe supérieur, respectivement trait discontinu et trait continu) et de la conductance du couvert $g_{c}$ (graphe inférieur). Les barres verticales représentent l'intervalle de confiance à $95 \%$ de la valeur moyenne estimée. A : 18 mai 1989; $B$ : 13 et 28 juin 1989 (journées de printemps, respectivement ensoleillées et couvertes); $C: 29$ juillet 1988 et 21 juillet 1989 (journées ensoleillées, respectivement sur sol humide et sur sol sec). fortement à la surface foliaire développée par la molinie. Leur longueur totale est, en moyenne, de $400-500 \mathrm{~mm}$, contre 150 $\mathrm{mm}$ pour la $1^{\text {re }}$ feuille. L'estimation de l'indice foliaire est sujette à une erreur importante liée à la variabilité spatiale de la biomasse par $\mathrm{m}^{2}$.

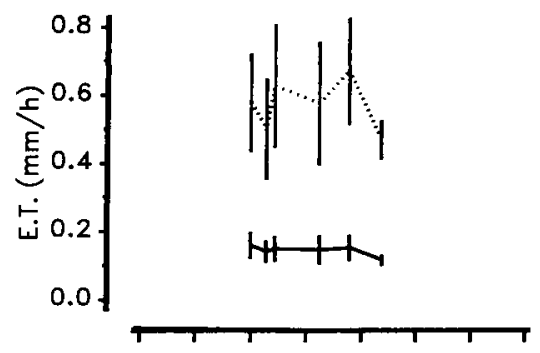

A

18 Mai 1989

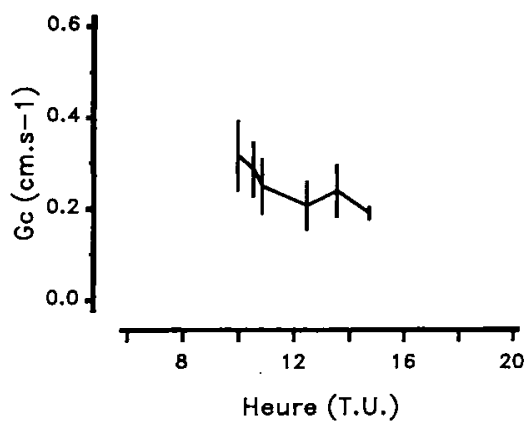




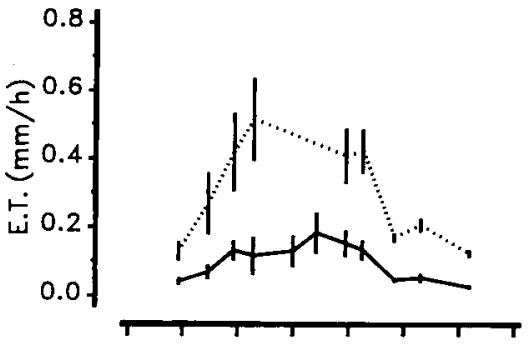

13 Juin 1989
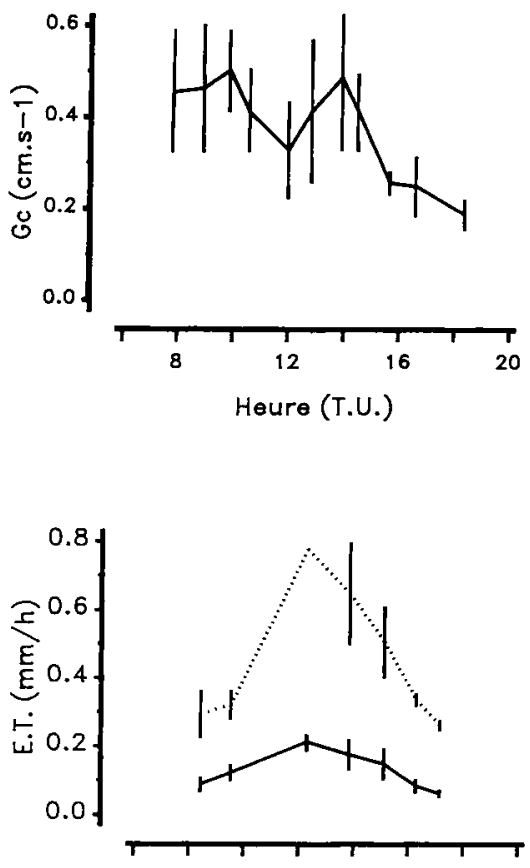

28 Juillet 1989

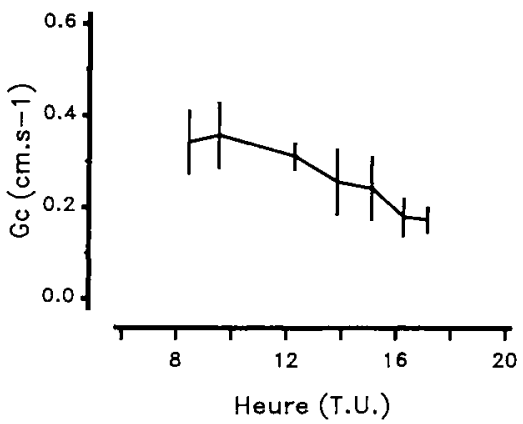

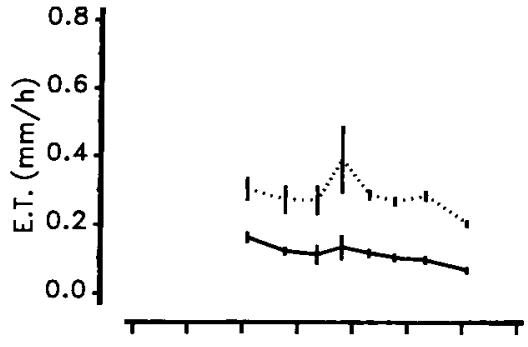

28 Juin 1989

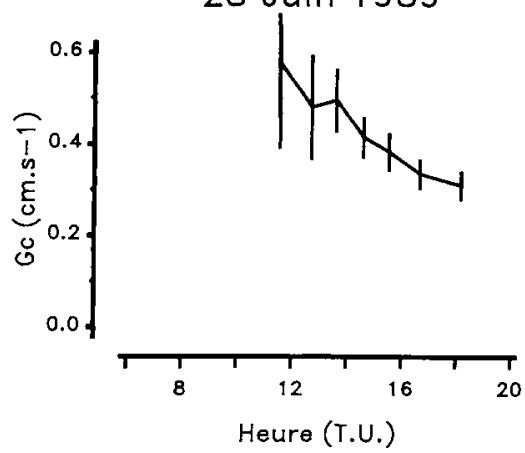

B

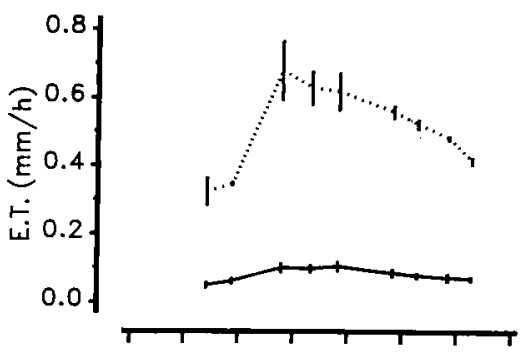

21 Juillet 1989

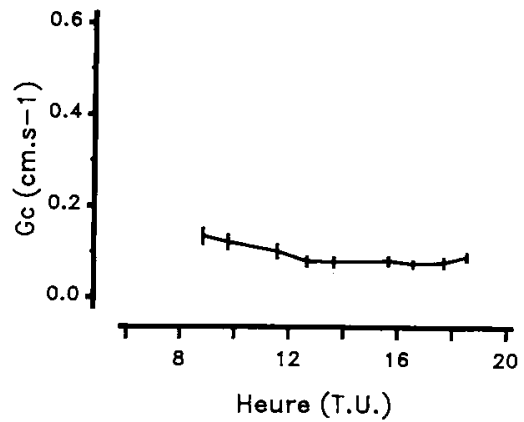

C 


\section{Évapotranspiration}

La figure 4 représente l'évolution journalière de l'ETP, de l'ET horaire et de la conductance de couvert du sous-bois au cours de 5 journées extraites des résultats obtenus en 1988 et en 1989. Les taux d'évapotranspiration horaires mesurés pour le sous-étage varient entre 0,05 et $0,25 \mathrm{~mm} \cdot \mathrm{h}^{-1}$. En début de saison, quand le sol est bien pourvu en eau, l'évolution journalière de l'ET suit globalement celle de l'ETP calculée à $1 \mathrm{~m}$. Cette relation $E T$ ETP s'observe jusqu'au mois de septembre en 1988 et jusqu'à la mi-juillet en 1989.

Les mesures les plus précoces dans la saison ont été faites au moment de la $2^{\theta}$ feuille (18 mai). Les valeurs de $g_{c}$ sont alors proches de $0,2 \mathrm{~cm} \cdot \mathrm{s}^{-1}$. Lors des journées suivantes, $g_{c}$ atteint $0,5-0,6 \mathrm{~cm} . \mathrm{s}^{-1}$ (août 1988 et juin-juillet 1989). En cours de journée, les valeurs les plus élevées de $g_{c}$ sont mesurées le matin, $g_{c}$ diminue ensuite progressivement jusqu'au soir. La variabilité spatiale de $g_{c}$, exprimée par l'erreur-standard à la moyenne (calculée pour les 10 touffes considérées) est relativement élevée.

Sur sol en phase de déssèchement, le taux d'ET horaire devient $\leq 0,1 \mathrm{~mm} \cdot \mathrm{h}^{-1}$ et varie relativement peu au cours de la journée. La figure $4 \mathrm{C}$ présente en vis-à-vis une journée de mesure de 1988 et une de 1989 , réalisées à la même période et présentant globablement le même profil d'ETP, mais différant par le niveau des réserves hydriques du sol. Sur sol sec, en 1989, le taux d'ET horaire est globalement réduit de moitié par rapport au taux mesuré sur sol humide en 1989.

Les valeurs de conductance chutent autour de $0,1 \mathrm{~cm} . \mathrm{s}^{-1}$ et restent stables au cours de la journée. La variabilité spatiale de $g_{c}$ est très atténuée par rapport à la période précédente.
La figure 5 représente les valeurs du taux d'ET horaire en fonction de celles de l'ETP à $1 \mathrm{~m}$, pour les journées de juin à octobre. Les journées où le stock hydrique du sol entre 0 et $75 \mathrm{~cm}$ est inférieur à 40 $\mathrm{mm}$ présentent une relation faiblement croissante entre le taux d'ET et l'ETP $\left(\mathrm{mm} . \mathrm{h}^{-1}\right)$. Cette relation peut s'écrire sous forme d'une régression linéaire passant par l'origine :

$$
E T=0,15 . E T P\left(d d l=32 ; r^{2}=0,94\right) \text {. }
$$

En revanche, quand le stock hydrique du sol est supérieur à $40 \mathrm{~mm}$, I'ET du sous-bois augmente plus fortement en fonction de l'ETP. La relation ET-ETP s'écrit alors :

$$
E T=0,29 . E T P\left(d d l=87 ; r^{2}=0,96\right) .
$$

Le tableau II représente les valeurs d'ET journalières du sous-étage et des arbres, calculées pour les 13 journées de mesure. L'ET du sous-étage représente 22-35\% de l'ETP mesurée au-dessus des arbres et correspond globabement à 1/3 de la transpiration des arbres quand le sol est bien pourvu en eau. Au cours de l'été 1989, qui fut exceptionnellement sec, l'ET journalière du sous-étage diminue et ne représente plus que $12-14 \%$ de l'ETP. La transpiration des arbres fut réduite de

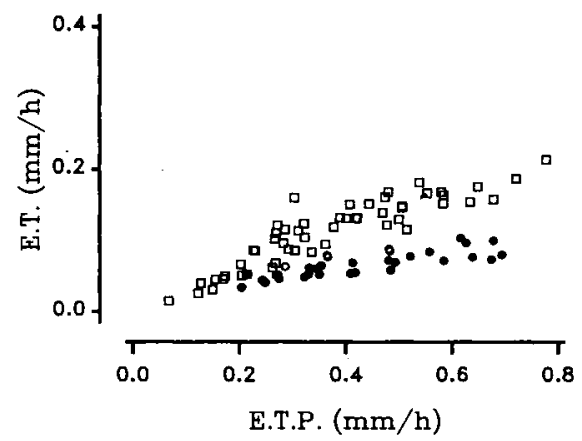

Fig 5. Relation entre l'ET du sous-étage de molinie et de I'ETP à $1 \mathrm{~m}$. $\square$ : stock hydrique supérieur à $40 \mathrm{~mm}$; : stock hydrique inférieur à 40 $\mathrm{mm}$. 


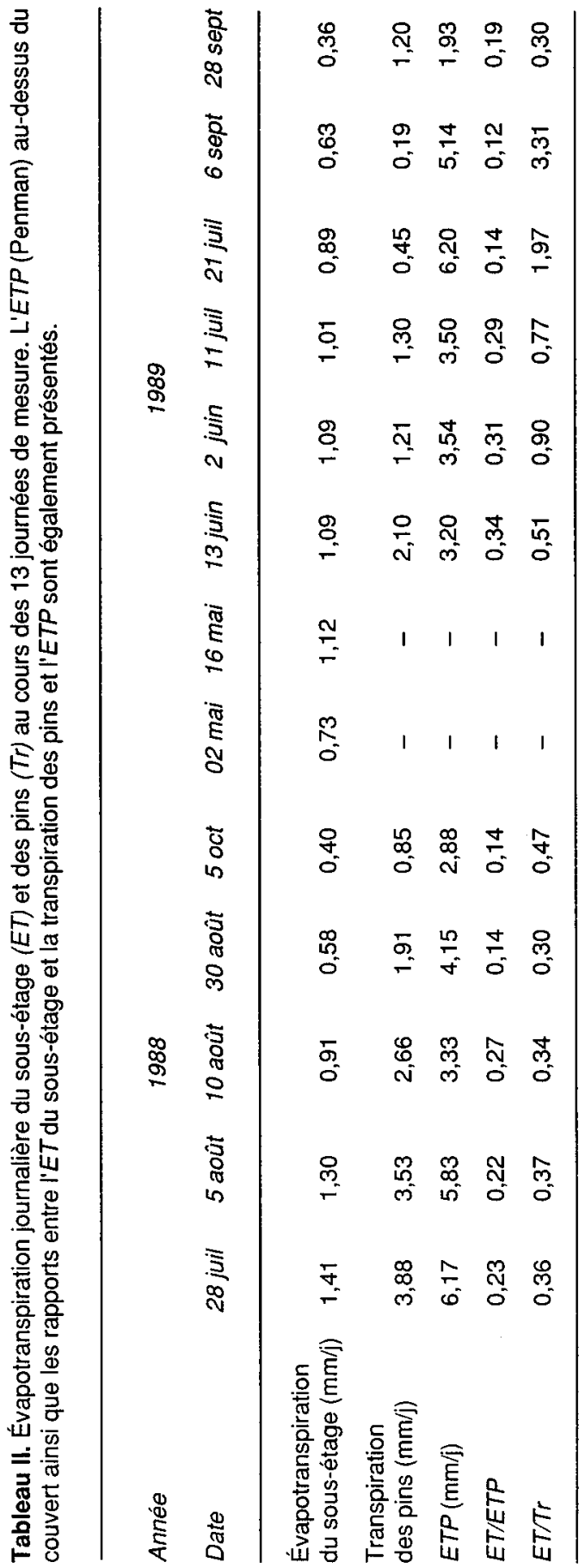


façon plus importante, d'un facteur 4 et plus, durant le développement de la sécheresse édaphique. Dans ces conditions l'ET du sous-étage lui devient supérieure.

\section{Conductance de couvert}

La figure 6 représente les valeurs de $g_{c}$ mesurées sur sol bien pourvu en eau et à indice foliaire supérieur à 1 , en fonction du déficit de saturation de l'air, pour 4 classes d'irradiance énergétique. Pour des déficits de saturation en vapeur d'eau de l'air excédant $3 \mathrm{kPa}$, la conductance de couvert ne dépasse jamais $0,2 \mathrm{~cm} . \mathrm{s}^{-1}$. Les valeurs de $g_{c}$ mesurées sous de faibles irradiances sont globalement inférieures à celles mesurées sous de fortes irradiances.

La figure 7 montre qu'il n'apparaît pas de relation nette entre l'indice foliaire d'une touffe et sa conductance, quelles que soient les conditions de disponibilité en eau du sol.

La figure 8 montre que la conductance de couvert maximale journalière, définie

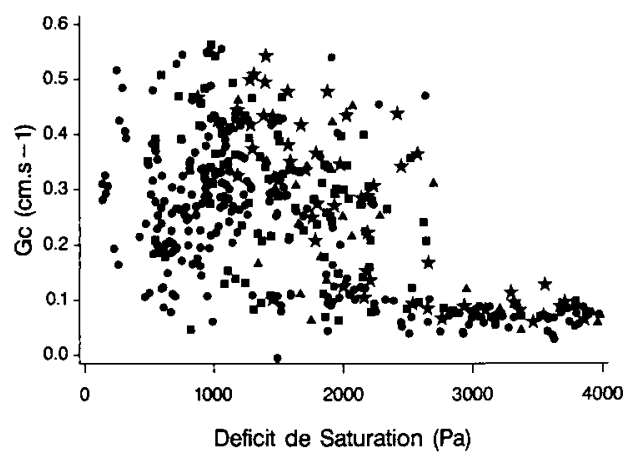

Fig 6. Diagramme des valeurs de conductance de surface $\left(g_{\delta}\right)$, en fonction du déficit de saturation de l'air pour 4 classes d'irradiance énergétiques: 075 W.m-2; ${ }^{-2}$ 75-150 W.m $\mathrm{m}^{-2}$; $\Delta$ 150$225 \mathrm{~W} \cdot \mathrm{m}^{-2} ; \star>250 \mathrm{~W} \cdot \mathrm{m}^{-2}$. Les mesures présentées ont été faites pour des indices foliaires supérieurs à 1 .

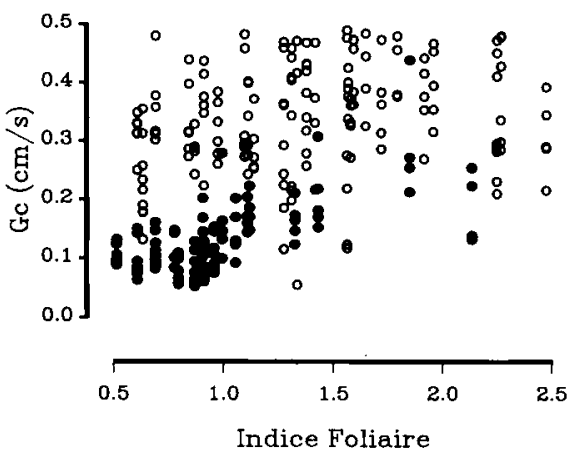

Fig 7. Relation entre l'indice foliaire d'une touffe et la conductance de couvert $O$ : stock hydrique du sol $(0-75 \mathrm{~cm})$ supérieur à $40 \mathrm{~mm} ;$ : stock hydrique du sol inférieur à $40 \mathrm{~mm}$.

comme la moyenne des valeurs de $g_{c}$ mesurées avant $11 \mathrm{~h} 00 \mathrm{TU}$, diminue quand le stock hydrique diminue en dessous de $40 \mathrm{~mm}$, sauf pour 2 journées. Ces 2 journées présentent cependant la particularité de succéder à des pluies de 6-8 $\mathrm{mm}$, intervenues la veille des mesures.

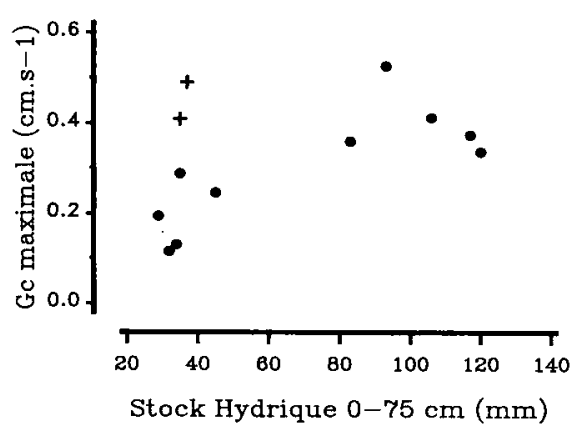

Fig 8. Diagramme des valeurs de conductance de surface maximale journalière en fonction du stock hydrique du sol $(0-75 \mathrm{~cm})$. Les croix $(+)$ correspondent à des journées succédant à de faibles pluies. 


\section{DISCUSSION}

Les successions phénologiques observées en 1988 et 1989 correspondent aux observations locales connues sur cette espèce (Bergeret, 1980). Contrairement à Jeffries (1915) et Loach (1968) en GrandeBretagne, nous n'avons pas observé le développement d'une $2^{\mathrm{e}}$ série de feuilles après l'épiaison. Dans l'Est de la France, Becker (1973) n'observe pas non plus ce développement végétatif tardif. Dans notre site expérimental, cette absence peut être mise en relation avec le développement d'une sécheresse édaphique estivale. La surface foliaire développée par la molinie en sous-bois ne devient importante qu'à partir du mois de mai; auparavant, la contribution de cette espèce à l'évapotranspiration du sous-bois est négligeable, et la plus grande partie du flux de vapeur du sous-étage est émise par évaporation. Les valeurs maximales d'indice foliaire sont atteintes en 1988 et en 1989 vers le début du mois de juillet et correspondent à une biomasse de $900 \mathrm{~kg} / \mathrm{ha}$ de matière sèche, relativement faible par rapport aux observations de Lemoine et al (1983), dans un contexte similaire.

\section{L'ET du sous-étage}

Elle comprend 2 composantes :

- l'évaporation à partir de la couche superficielle du sol,

- la transpiration des feuilles de molinie.

\section{Évaporation du sol}

En conditions naturelles, l'évaporation à partir de la litière sous un couvert herbacé d'indice foliaire supérieur à 1 est sans aucun doute négligeable. Kelliher et al (1986), dans des conditions expérimentales voisines des nôtres, donnent une es- timation de la conductance à la diffusion de vapeur de la couche superficielle d'un sol de $0,125 \mathrm{~cm} . \mathrm{s}^{-1}$ pour une humidité volumique moyenne du sol supérieure à $18 \%$. Pour des humidités volumiques plus faibles, la conductance du sol à la diffusion diminue en fonction de l'humidité volumique, suivant la relation :

$$
g_{0}=(-830 . \theta+161)^{-1}
$$

$g_{0}=$ conductance à la diffusion du sol (cm.s ${ }^{-1}$ )

$\theta=$ humidité volumique moyenne sur la profondeur de sol utile $\left(\mathrm{cm}^{3} \cdot \mathrm{cm}^{-3}\right)$.

En reprenant cette estimation, il est ainsi possible de calculer par approximation le taux d'évaporation du sol dans la chambre (équation 1). Pour une humidité volumique moyenne supérieure à $18 \%$, qui correspond à un sol légèrement en dessous de la capacité au champ, et pour un déficit de saturation de $2 \mathrm{kPa}$, ce taux serait de $0,06 \mathrm{~mm} / \mathrm{h}$. Dès que l'humidité volumique moyenne du sol diminue, ce taux chute rapidement. Pour une humidité volumique de $15 \%$, il passerait à $0,015 \mathrm{~mm} / \mathrm{h}$, ce qui représente le plus souvent une fraction négligeable de l'ET totale du sousétage.

Cette estimation montre que l'évaporation à partir du sol nu peut correspondre à une fraction importante de l'ET du sousétage quand le sol est proche de la saturation. C'est le cas pour certaines journées de mesures du printemps, 2 mai et 18 mai 1989), où l'indice folaire est encore inférieur à 1 (fig 3); la participation de l'évaporation du sol à l'ET totale mesurée n'est sans doute pas négligeable. En revanche, elle deviendrait minime dès que l'humidité volumique du sol descend en dessous de $15-16 \%$. Ce seuil est atteint vers la miaoût en 1988 (6 août et au-delà) et dès la fin juin en 1989 (28 juin et au-delà). Les valeurs d'ET mesurées ultérieurement expriment donc principalement la transpiration de la molinie. 


\section{Transpiration de la molinie}

La mesure d'un taux de transpiration par une méthode de type chambre close peut être biaisée par la ventilation forcée qui augmente la conductance de la couche limite de la surface évaporante (Rose et Sharma, 1984). Cette surestimation pourrait atteindre $15 \%$ de la valeur d'évapotranspiration mesurée (Cochard, 1988) mais nous n'avons pas effectué de mesures précises de cette erreur.

Ceci conduit le plus souvent à restreindre la portée des résultats de mesures d'ET avec une chambre de transpiration, à un aspect comparatif.

Les taux horaires de transpiration du sous-étage de molinie sont élevés si on les compare avec ceux obtenus pour un sous-bois de pin sylvestre à fougères (Roberts et al, 1980) ou à éricacées (Lindroth, 1984) ou aux valeurs données par Kelliher et al (1986) pour un sous-étage de douglas à éricacées. Ces écarts s'expliquent par les différences climatiques entre les différents sites expérimentaux et par la composition spécifique du sous-étage dans les différents peuplements étudiés. Le rapport entre l'évapotranspiration du sous-étage et la transpiration des arbres reste cependant comparable aux valeurs données par Roberts et al (1980) et Black et al (1980), et confirment les estimations de Granier et al (1990) pour un sous-bois de pin maritime à fougères.

La sensibilité de l'évapotranspiration du sous-étage au niveau de la réserve hydrique du sol (fig 5) et l'augmentation de sa participation à l'ET totale au cours du dessèchement édaphique (tableau II) sont aussi observées par Roberts et al (1980). Dans notre cas, l'ET journalière du sousétage peut représenter jusqu'à plus de 2 fois celle des arbres (fin d'été 1989) comme cela est observé par Tan et al (1978) dans un peuplement de douglas.
Cet écart important traduit à la fois l'importance de la réduction de la transpiration des arbres (Loustau et al, 1990) et le maintien de l'ET du sous-étage à un niveau relativement élevé $\left(0,6 \mathrm{~mm} . \mathrm{j}^{-1}\right)$. Une étude des variations saisonnières et journalières du bilan d'énergie dans ce peuplement (Berbigier et al, 1990) conduit à des conclusions identiques sur l'évapotranspiration respective de chacune de ces 2 strates pendant cette période.

\section{Conductance de couvert}

Compte tenu de l'approximation que nous avons opérée sur la valeur de $g_{a}$, la conductance de couvert calculée intègre en fait une composante de surface au sens strict et une composante de couche limite résiduelle, inconnue. $A$ indice foliaire égal, cette composante résiduelle est constante et identique pour toutes les touffes mesurées, ce qui permet d'interpréter globalement les variations de la conductance de couvert comme des modifications de la seule composante de surface. Celles-ci traduisent les modifications des caractéristiques du transfert de vapeur entre le mésophylle des feuilles de molinie et l'atmosphère.

Cependant, les écarts entre la température de surface des feuilles et celle de l'air peuvent induire des erreurs importantes dans le calcul de $g_{c}$. La possibilité d'opérer une mesure fiable d'une température moyenne de surface d'un ensemble de végétation comme une touffe de feuilles de molinie pourrait réduire cette erreur. Actuellement, en l'absence de comparaison précise avec d'autres méthodes de mesure de la conductance de couvert de ce sousbois, nos résultats et leur interprétation sur ce point doivent donc être considérés avec prudence. 
Les valeurs relativement faibles de conductance du couvert mesurées au moins de mai pourraient être liées au faible indice foliaire $(0,7)$ de la molinie à ce moment, ou à une conductance plus faible des premières feuilles. Celles-ci représentent une proportion notable de l'indice foliaire à ce moment.

Les conductances élevées mesurées au mois de juin sont sans doute légèrement surestimées, l'évaporation du sol pouvant représenter une fraction notable de I'ET mesurée. La conductance de couvert semble presque insensible à l'irradiance énergétique ou au déficit de saturation de l'air tant que celui-ci ne dépasse pas $3 \mathrm{kPa}$ (fig 6 et 7), et elle varie peu au cours de la journée. Guinochet et Lemée (1950) observent une fermeture stomatique chez la molinie, en condition d'évaporation intense. Ce mécanisme pourrait expliquer la limitation de la conductance de couvert pour ces déficits de saturation de l'air élevés. En dehors de cette limitation, les conditions microclimatiques des mesures correspondent probablement à une ouverture stomatique maximale des feuilles de molinie. La conductance de couvert semble peu sensible à l'indice foliaire des touffes mesurées (fig 7). Ceci suggère que la transpiration moyenne par unité de surface diminue quand l'indice foliaire de la touffe augmente. La conductance de la couche limite des feuilles de la partie basale d'une touffe, serrées les unes contre les autres, pourrait être plus faible que celle des feuilles de la partie sommitale, qui sont moins denses et plus étalées. Les plus grosses touffes présentent proportionnellement plus de feuilles en position basale que les autres. Cet effet pourrait donc expliquer en partie l'absence d'une relation entre $g_{c}$ et l'indice foliaire.

Les conditions de disponibilité en eau du sol pour la molinie ne sont qu'imparfaitement exprimées par le niveau global des réserves hydriques du sol. Des précipitations de quelques $\mathrm{mm}$ peuvent recharger la couche superficielle du sol et restaurer momentanément une certaine disponibilité de l'eau pour la molinie. Ce phénomène pourrait expliquer les valeurs élevées de maxima journaliers de conductance de couvert mesurés pour des stocks inférieurs à $40 \mathrm{~mm}$ (fig 8).

La diminution de la conductance de couvert pour un stock hydrique édaphique inférieur à $40 \mathrm{~mm}$ (fig 8) peut s'expliquer par 2 mécanismes : la fermeture stomatique, qui diminue la composante de surface de la conductance du couvert, et l'enroulement des feuilles, observé en 1988 comme en 1989, qui réduit la surface foliaire exposée et diminue la conductance de la couche limite de la face supérieure, concave, des feuilles.

Bien que Guinochet et Lemée (1950) aient observé une fermeture stomatique rapide pour des feuilles de molinie soumise à une sécheresse édaphique, ces auteurs soulignent les effets limités de ce mécanisme sur la transpiration, qu'ils attribuent à l'existence d'une transpiration cuticulaire élevée.

L'enroulement des feuilles de graminées peut réduire la transpiration d'un facteur 3 (Renard et Demessemacker, 1983). La face supérieure des feuilles de molinie présente une densité stomatique de 200250 stomates $/ \mathrm{mm}^{-2}$ contre $40-50$ pour la face inférieure. L'efficacité de l'enroulement des feuilles pour réduire la transpiration est donc potentiellement importante.

Ces réactions de la plante semblent réduire la variabilité de la conductance de couvert entre les touffes de molinie d'une part (fig 4c) et entre les feuilles d'une même touffe d'autre part.

Bien que les mesures d'évapotranspiration et de conductance de couvert à partir de méthodes micrométéorologiques n'aient 
pas été faites durant les mêmes journées que celles opérées avec la chambre de transpiration, elles conduisent à des résultats similaires en ce qui concerne les évolutions journalière et saisonnière des valeurs de $g_{c}$ (Berbigier et al, 1990).

\section{CONCLUSION}

Les limites de l'utilisation de la chambre de transpiration utilisée sont surtout liées à la possibilité d'estimer la conductance de la couche limite du matériel mesuré et d'en mesurer la température de surface. Ceci permettrait d'éviter les biais induits par la surventilation sur l'ET mesurée d'une part et d'améliorer la précision obtenue sur le calcul de $g_{c}$ d'autre part.

Actuellement, cette méthode conduit à des résultats comparables à ceux obtenus à partir d'autres méthodes (Berbigier et al, 1990 ) et permet une analyse des variations saisonnières de la conductance de couvert du sous-bois. L'évapotranspiration du sous-bois de molinie pendant la saison de végétation représente $1 / 3$ de celle des arbres. Elle se montre moins sensible que celle-ci à la sécheresse édaphique estivale. La réponse de la conductance de couvert du sous-bois aux facteurs du milieu semble aussi très atténuée comparativement à celle des arbres (Loustau et Granier, 1991). Ces conclusions suggèrent l'intérêt potentiel d'un contrôle de la végétation du sous-bois pour retarder la sécheresse estivale et en réduire l'intensité.

\section{REMERCIEMENTS}

Les auteurs remercient le $\operatorname{Pr} B$ Saugier et JY Pontailler (Laboratoire d'écologie végétale de l'université d'Orsay), ainsi que A Granier (station de sylviculture et production, INRA Nancy),
C Riou et $C$ Valancogne (station de bioclimatologie, INRA Bordeaux), qui les ont fait bénéficier de leurs conseils pour la mise au point de la chambre de transpiration et des systèmes de mesures météorologiques de la parcelle. Les auteurs remercient également $A$ Elhabchi qui a largement contribué aux mesures effectuées en 1989 durant son stage de DEA (université de Pau) et Madame Guillem, qui a effectué la saisie du texte de cet article.

Ce travail a été effectué grâce aux aides de I'ATP Piren «Influence à l'échelle régionale des couvertures végétales et pédologiques sur les bilans hydriques et minéraux du sol» et de l'AlP "Fonctionnement hydrique du pin maritime". Durant son stage de DEA, $H$ Cochard a bénéficié d'une bourse de l'INRA.

\section{RÉFÉRENCES}

Amadou M (1989) Estimation de l'évapotranspiration d'un couvert de pin maritime (Pinus pinaster Ait), par la méthode du bilan d'énergie - fluctuations de température et de vitesse verticale de vent. Rapport de stage de DAA ENSA de Rennes, sept 1989

Becker M (1973) Contribution à l'étude expérimentale de l'écologie de cinq espèces herbacées forestières. CEcol Plant 8, 99-124

Berbigier P, Diawara A, Loustau D (1990) Étude microclimatique de l'effet de la sécheresse sur l'évaporation d'une plantation de pins maritimes et du sous-bois. Communication au Colloque Effets physiologiques de stress du Groupe d'étude de l'arbre, Nancy, 27-28 mars 1990

Bergeret H (1980) Évolution floristique et production de la lande à molinie (Molinia coerulea (L) Moench) en forêt landaise. Thèse de $3^{\theta}$ cycle, Univ Bordeaux III

Black TA, Tan CS, Nnyamah U (1980) Transpiration rate of Douglas-fir trees in thinned and unthinned stands. Can J Soil Sci 60, 625-631

Cochard $H$ (1988) Utilisation d'un système de type "enceinte fermée» pour l'estimation de la transpiration journalière d'un sous-bois de Pin maritime (Pinus pinaster Ait). Rapport de stage de DEA - Univ Orsay, oct 1988

Cruiziat P, Thomas DA, Bodet C (1979) Comparaison entre mesures locales et mesures glo- 
bales de la résistance stomatique des feuilles de Tournesol (Helianthus annuus). CEcol Plant 14, 447-459

Daudet A (1987) Un système simple pour la mesure in situ des échanges gazeux de couverts végétaux de quelques mètres carrés de surface foliaire. Agronomie 7, 133-139

Decker JP, Gaylor WG, Cole FD (1962) Measuring transpiration of undisturbed Tamarisk shrubs. Plant Physiol 37, 393-397

Diawara A, Loustau D, Berbigier P (1990) Comparison of two methods for estimating the evaporation of a Pinus pinaster (Ait) stand : sap flow and energy balance with sensible heat flux mesurements by an eddy covariance method. Agric For Meteor (sous presse)

Granier A (1987) Evaluation of transpiration in a Douglas fir stand by means of sap-flow measurements. Tree Physio/ 3, 309-320

Granier A, Bobay V, Gash JHC, Gelpe J, Saugier B, Shuttleworth WJ (1990) Sap flow measurements of transpiration from a stand of maritime Pine (Pinus pinaster Ait) in Les Landes Forest. Agric For Meteor (sous presse)

Guinochet, Lemée (1950) Contribution à la connaissance des races biologiques de Molinia cœrulea (L) Moench. Rev Gén Bot 680, 563-593

Jefferies TA (1915) Ecology of the Purple Heath Grass (Molinia coerulea). J Ecol 3, 93-109

Jones HJ (1983) Plants and Microlimate, Cambridge Univ Press

Kelliher FM, Black A, Price DT (1986) Estimating the effects of understory removal from a Douglas fir forest using a two-layer canopy evapotranspiration model. Water Resour Res 22, 1891-1899

Landsberg JJ, Powell DBB (1973) Surface exchange characteristics of leaves subject to mutual interference. Agric Meteorol 12, 169184

Lemoine B, Bonhomme D, Chinzi D, Comps B, Bergeret $H$, Gelpe J, Juste $C$, Menet M (1983) Élevage en forêt dans les Landes de
Gascogne. 1- Le système végétal. Ann Sci For 40, 3-40

Lindroth A (1984) Seasonal variation in Pine Forest - Evaporation and Canopy conductance. Acta Univ Ups Abstr Ups Diss Sci 758, 1984

Loach K (1968) Seasonal growth and nutrient uptake in a Molinietum. $J$ Eco/ $56,433-444$

Loustau D, Granier A (1991) Environmental control of water flux through maritime Pine. In: Analysis of Water Transport in Plants and Cavitation of Xylem Conduits (Raschi A, Borghetti M, Fantechi R, Grace J, eds) Proceedings of the Vallombrosa Int Workshop, Firenze, Italy. 29-31 May 1990 (à paraître)

Loustau D, Granier A, El Hadj Moussa F (1990) Évolution saisonnière du flux de sève dans un peuplement de pins maritimes. Ann Sci For 47, 599-618

Murray FW (1967) On the computation of saturation vapour pressure. $J$ Appl Meteor 6, 203204

Peters DB, Clough BF, Garves RA, Stahl GR (1974) Measurement of dark respiration, evaporation, and photosynthesis in field plots. Agron J 66, 460-462

Reicosky DC, Peters DB (1977) A portable chamber for rapid evapotranspiration measurements on field plots. Agron J 69, 729-732

Renard C, Demessemacker W (1983) Effects of wind velocity on stomatal conductance and consequences of leaf rolling on water uptake in tall Fescue. Biol Plant 25, 408-411

Roberts J, Pymar CF, Wallace JS, Pitman RM (1980) Seasonal changes in leaf area, stomatal and canopy conductances and transpiration from bracken below a forest canopy. $J$ Appl Ecol 17, 409-422

Rose CW, Sharma ML (1984) Summary and recommendations of the workshop on "Evapotranspiration from plant communities". Agric Water Manag 8, 325-342

Tan CS, Black TA, Nnyamah JU (1978) A simple diffusion model of transpiration applied to a thinned Douglas fir stand. Ecology $59,1221-1229$ 